45

46

47

48

\section{Exposing avian embryos to light affects post-hatch anti-predator fear responses} Gregory S. Archer* and Joy A. Mench

\author{
Department of Animal Science, University of California, Davis
}

*Corresponding Author

Poultry Science Department

101 Kleberg Building

MS 2472 TAMU

College Station, TX 77845

garcher@poultry.tamu.edu 
50 Environmental stimuli present during incubation can impact the behavior of birds

51 post-hatch. To determine the effect of exposing broiler chicken embryos to light on fear-

52 related behavior post-hatch, we conducted two experiments in which we incubated eggs

53 under various light schedules, and then measured fear responses when the chickens $(\mathrm{N}=$

54 720) were 3-6 wk of age. In Expt. 1, the incubation photoperiods were 0L:24D, 12L:12D,

55 and 24L:0D, and tonic immobility (TI) and inversion (INV) tests were administered. In

56 Expt. 2, the incubation photoperiods were 0L:24D, 1L:23D, 6L:18D, and 12L:12D; and

57 an approach test (APPR) and an emergence (EMRG) test were administered in addition

58 to TI and INV. In Expt. 1, both 12L:12D and 24L:0D had shorter latencies to right

59 during TI (213.5 \pm 23.7 and $231.8 \pm 24.2$ sec, respectively) than 0L:24D (305.5 \pm 26.1

$60 \mathrm{sec})$ and also wing flapped less intensely during INV (12L:12D $5.0 \pm 0.1$ wing flaps;

61 24L:0D $5.4 \pm 0.2)$ than 0L:24D (5.7 \pm 0.1$)$. In Expt. 2, the 12L:12D birds once again had

62 shorter latencies to right during TI (120.0 $\pm 16.5 \mathrm{sec})$ and wing flapped less intensely

63 during INV (4.7 \pm 0.1 wing flaps) than 0L:24D (201.2 \pm 24.9 and $5.5 \pm 0.1$, respectively).

64 They also had shorter latencies to exit the dark box in EMRG (28.9 \pm 3.3 seconds), and

65 were less active (28 $\pm 2 \%$ ), vocalized less (178.8 \pm 9.3 times/3min) and spent more time

66 closer to the observer during APPR $(63 \pm 3 \%)$ than $0 \mathrm{~L}: 12 \mathrm{D}(42.9 \pm 5.0$ seconds, $35 \pm 3 \%$,

$67211.2 \pm 10.4$ times/3 min, $51 \pm 3$ ). The 1L:23D and 6L:18D showed some reductions in

68 fearfulness compared to 0L:24D, but these were not consistent across tests. The 6L:18D

69 and 12L:12D birds demonstrated lateralization in the direction to leave the box in EMRG,

70 whereas 1L:23D and 0L:12D exited left or right at chance levels. The results of these

71 experiments indicate that providing at 12 hours of light stimulation daily g 
72 embryogenesis results in long-term reductions in fearfulness as measured by multiple

73 tests, and that this may be related to cerebral lateralization. In conjunction with other

74 research, these findings show that light exposure during embryogenesis has important

75 implications for behavioral phenotypes and welfare in chickens.

76

77 Keywords: light, avian, embryo, fear, incubation, lateralization

78

79 


\section{Introduction}

81 Avian behavioral phenotypes are influenced by both the internal and external

82 environment during embryogenesis. Maternal deposition of androgens into the eggs can

83 alter offspring phenotypes (e.g. related to boldness or alertness) such that the offspring

84 are better suited for current environmental conditions (Groothuis et al., 2005). Avian

85 embryos also respond to external stimuli such as olfactory, auditory and photoperiodic

86 cues (Reed and Clark, 2011). For example, exposure to species specific call during

87 embryonic development is important for post-hatch species recognition in some avian

88 species (Gottlieb, 1976, 1985). Olfactory stimulation can also shape later behavior.

89 Bertin et al. (2010) observed that chickens exposed to odors during incubation preferred

90 food bearing those odors post-hatch.

91 Photoperiodic cues are particularly important factors influencing post-hatch

92 phenotypes. Embryos receive regular brief light exposures when their parent(s) leave the

93 nest to feed (Buschmann et al., 2006; Mrosovsky and Sherry, 1980) or respond to distress

94 calls from the embryos by rising to turn the eggs (Rogers, 1996). This light can penetrate

95 the eggshell and reach the embryo where it is sensed either by developing retinal cells or

96 the pineal gland (Cooper et al., 2011), resulting in melatonin synthesis. Melatonin affects

97 embryonic growth rates as well as the development of the visual, skeletal and immune

98 systems (see Reed and Clark, 2011).

99 Prenatal light exposure has also been shown to affect post-hatch behavior.

100 Domestic fowl chicks exposed to light during incubation have a different diurnal rhythm

101 of feeding activity than dark-incubated chicks (Archer et al., 2009), and also differ from

102 dark-incubated chicks in terms of discrimination learning ability (Rogers, 1990), memory 
103 retention for a passive avoidance task (Sui and Rose, 1997), and aspects of social

104 behavior including social exploration, social recognition, and competitive success

105 (Riedstra and Groothuis, 2004; Rogers and Workman, 1989). Chicks exposed to light

106 during incubation also show more avoidance of an unfamiliar imprinting object than

107 dark-incubated chicks during the first 12 hours post-hatch (Dimond, 1968).

108 The mechanisms underlying the behavioral effects of light stimulation during

109 incubation in birds probably involve both melatonin effects on behavioral rhythms and

110 the direct effect of embryonic light stimulation on brain development via lateralization of

111 the thalamofugual and tectofugal visual pathways (Rogers 1982). The latter results in

112 hemispheric specialization, with the left hemisphere specialized for visual discrimination

113 tasks, food-searching and vocal production and recognition, while the right is specialized

114 for situations that involve a strong negative affective component, such as fear or

115 aggression (Phillips and Youngren, 1986; Rogers, 2008). It has been hypothesized that

116 strong lateralization is adaptive in that it facilitates dual processing of sensory

117 information, allowing individuals to efficiently perform two tasks simultaneously. This

118 hypothesis has been tested in domestic fowl (Dharmaretnam and Rogers, 2005) using

119 simultaneous presentations of food acquisition tasks and predator stimuli. These studies

120 showed that lateralized light-incubated chicks were able to more efficiently obtain food

121 while continuing to maintain vigilance than less lateralized individuals.

122 Despite the presumed adaptive value of the patterns of lateralization found in

123 light-incubated chicks, there is actually limited evidence that the behaviors observed at

124 young ages persist throughout development. Shifts in lateralization for fear behavior in

125 domestic chicks have been reported to occur between 5 and 15 days of age post-hatch 
126 (Andrew and Brennan, 1983), but the effects of providing light during incubation on

127 lateralization and fear responses in older birds have not been evaluated.

$128 \quad$ Our previous studies suggest that providing light during incubation can have

129 beneficial effects on the welfare of broiler chickens 3-6 weeks post-hatch (Archer et al.,

130 2009; Archer and Mench, 2009), decreasing composite asymmetry, reducing the

131 corticosterone response to crating stress, and enhancing antibody response to challenge.

132 We conducted two experiments to evaluate whether providing light during incubation

133 also had developmentally persistent effects on fearfulness. Since anti-predator responses

134 have been shown to be the most reliable measures for assessing fear (Miller et al., 2005,

135 2006), we utilized four different fear tests involving anti-predator responses (inversion,

136 tonic immobility, emergence, and approach). We also assessed the degree of laterality in

137 light and dark-incubated chicks by determining the direction of movement in the

138 emergence test.

139

140 2. Materials and methods

141 2.1. Animals and husbandry

142 We obtained fertilized chicken eggs (Cobb 500 strain) from a commercial

143 hatchery, and randomly assigned them to be incubated under different lighting schedules.

144 In Experiment $1(\mathrm{~N}=664$ eggs) we used three lighting schedules: 0 hours of light and 24

145 hours of darkness (0L:24D), 24 hours of light and 0 hours of darkness (24L:0D), or 12

146 hours of light and 12 hours of darkness (12L:12D). In Experiment 2 ( $\mathrm{N}=1512$ eggs) we

147 used four lighting schedules: 0 hours of light and 24 hours of darkness (0L:24D), 1 hour

148 of light and 23 hours of darkness (1L:23D), 6 hours of light and 18 hours of darkness 
149 (6L:18D), or 12 hours of light and 12 hours of darkness (12L:12D). The lighting

150 durations in Experiment 1 were used to determine if there was an effect of light at the

151 extremes (constant or no light) and/or the mid-point. Experiment 2 used shorter lighting

152 durations to determine if there was a minimal amount of light needed during incubation

153 to obtain the results observed in Experiment 1. The intensity of light at the egg level

154 within the incubators was 550 lux as measured with a photoreceptor sensor of a light

155 meter (LT Lutron, model LX-100M; Das Distribution Inc., East Granby, CT). In

156 Experiment 1 lighting was controlled manually by covering or uncovering the clear

157 incubator top with cardboard to block any light entering, while in Experiment 2 each

158 treatment was contained within a ventilated environmentally controlled light-tight box

159 that allowed the photoperiod to be automatically controlled by timers. Temperature and

160 humidity inside the incubators (Hovabator; G.Q.F. Manufacturing Co., Savannah, GA)

161 were monitored to insure that the conditions in the different incubators were similar. We

162 candled eggs (Cool-Lite tester, GQF, Savannah, GA) once a week and removed non-

163 viable eggs. Each experiment was conducted in three trials, with three incubators per

164 treatment per trial.

165 Following completion of hatch all chicks were moved to a housing room at the

166 Hopkins Avian Research Facility at the University of California, Davis. They were

167 managed according to the guidelines set forth in the Guide for the Care and Use of

168 Agricultural Animals in Agricultural Research and Teaching (FASS, 2010). All

169 experimental procedures were approved by the Institutional Animal Care and Use

170 Committee of the University of California, Davis. 
172 Experiment 2) from each trial in one of nine pens (Experiment 1) or one of twelve pens

173 (Experiment 2) by incubator, with treatments randomized by block (incubator)

174 throughout the room. Each pen was $6.0 \mathrm{~m}^{2}$ and was bedded with wood shavings

175 (approximately $10 \mathrm{~cm}$ deep). Chicks were given access to feed and water ad libitum

176 throughout the study. They were fed a pre-starter mash (23.21\% crude protein,

177 3.10kcal/g metabolizable energy) for the first 3 weeks, and then Purina Mills Flock

178 Raiser Sunfresh Crumble ${ }^{\mathrm{TM}}$ (St. Louis, MI; 20\% protein, 3.00kcal/g metabolizable

179 energy) for the remainder of the 6 week experiment.

180 The birds were raised under a 12L:12D photoperiod with a photophase light

181 intensity of 250 lux. Light intensity was measured along a horizontal plane at $25 \mathrm{~cm}$

182 above the floor with the photoreceptor sensor of the light pointed toward the light

183 sources. There were no dawn-dusk transitions between the light and the dark phase of the

184 photoperiod. Light intensity during the dark phase was 0 lux (complete darkness).

\section{2.2. Fear measurements}

We used tonic immobility (TI) and inversion (INV) tests to measure anti-predator

189 responses in both experiments. Tonic immobility testing was carried out as described by

190 Archer and Mench (2014) on 10 randomly selected birds (5 males and 5 females;

191 determined by secondary sex characteristics which were confirmed at 5 weeks of age.)

192 per pen when the birds were five weeks old. In brief, we placed birds on their backs in a

193 wooden cradle and held them there for 15 seconds. If the bird righted before 10 seconds

194 TI was re-induced up to three times. If the bird could not be induced in three attempts it 
195 was scored as 0. We recorded latency to first head movement, latency to right, and

196 number of induction attempts. The test was terminated in 600 seconds if a bird failed to

197 right, and that bird was scored as 600.

198 When the birds were 42 days old we subjected the same ten birds that had been

199 used for TI testing to an INV test, as described by Newberry and Blair (1993) and Archer

200 and Mench (2014). We caught each bird and then inverted it by holding it by its legs

201 with one hand until the bird ceased to wing flap, or for 30 seconds. We determined the

202 duration of and number of wing flaps, the number of vocalizations, and the number of

203 body curls from video recordings (Cannon, ZR900, Melville, NY, USA; 24 frames per

204 second).

205 In Experiment 2 we administered two additional fear tests, the approach test

206 (APPR) and the emergence test (EMRG), when the birds were three weeks of age. Ten

207 randomly selected birds (5 males and 5 females) were selected per pen to be used in both

208 of these tests and in the TI and INV tests that followed. Both tests were videotaped

209 (Cannon, ZR900, Melville, NY, USA; 24 frames per second). For APPR we placed the

210 bird in a $1 \mathrm{~m} \times 0.5 \mathrm{~m} \times 0.6 \mathrm{~m}$ enclosure that had three solid sides made of wood and a front

211 made of chicken wire. The observer (GSA) then immediately sat approximately $0.5 \mathrm{~m}$

212 from the front of this enclosure, visible to the bird. The enclosure was painted black and

213 marked with white paint into three equal areas and the bird's proximity to the observer,

214 its activity (\% of time moving), and the number of vocalizations given (distress calls)

215 were recorded via video for three minutes after the bird was placed in the enclosure. The

216 enclosure was cleaned between birds. 
For EMRG we placed a bird in a wooden dark box $(0.3 \times 0.3 \mathrm{~m})$, via a hatch in the

218 top of the box, for thirty seconds. We then removed the front partition of the box so that

219 the bird could exit either left or right down one of two perpendicularly attached wooden

220 alleyways ( $0.6 \mathrm{~m}$ in length by $0.3 \mathrm{~m}$ height, covered with chicken wire). Latency to exit

221 the box was determined from video, with the camera placed out of the sight of the bird to

222 limit potential disturbances that would affect emergence. Directionality (Left $=-1$, Right

$223=1$ ) was recorded as a measure of laterality. Each bird was tested twice with a two day

224 period between testing. An average directionality score significantly different from zero

$225(\mathrm{P}<0.05)$ indicated that the directionality of exit was not at chance levels. The test

226 apparatus was cleaned between birds.

\subsection{Statistical analysis}

We used a one-way ANOVA to analyze treatment effects for the TI, INV, and 231 APPR tests, and a GLM with treatment, testing day, and their interaction to analyze the

232 EMRG test. We performed LSD post hoc tests comparing each light treatment group to

233 the controls (0L:24D) when we found significant differences. All data were tested to

234 ensure that they met the assumptions of ANOVA (Shapiro-Wilk test for normality,

235 Levene's test for homogeneity of variance); no transformations were required to meet

236 these assumptions. All analyses were performed using SAS 9.2 for Windows (SAS

237 Institute Inc.). We also used a T-test to determine whether directionality when exiting the

238 dark box in the EMRG test differed from chance (mean $=0, \mathrm{P}<0.05$ ).

\section{$239 \quad 3.0$ Results}

240 3.1. Tonic immobility 
242 right (Experiment 1: $\mathrm{F}_{2,177}=4.2, \mathrm{P}=0.02$; Experiment 2: $\mathrm{F}_{3,336}=4.37, \mathrm{P}=0.005$, Table

243 1). In Experiment 1, both the 12L:12D and 24L:0D birds had shorter latencies to right

244 than the $0 \mathrm{~L}: 24 \mathrm{D}$ birds $(\mathrm{P}=0.007$ and $\mathrm{P}=0.03$, respectively). The $0 \mathrm{~L}: 24 \mathrm{D}$ birds had the

245 longest latency to right in both experiments. Latency to first head movement and number

246 of induction attempts did not differ between treatments $(\mathrm{P}>0.05)$ in either experiment

247 (Table 2).

248 3.2. Inversion test

249 The INV response also differed between treatments in both studies (Experiment 1:

$250 \mathrm{~F}_{2,173}=5.87, \mathrm{P}=$ 0.003; Experiment 2: $\mathrm{F}_{3,336}=8.35, \mathrm{P}<0.001$, Table 1). In Experiment

251 1, the 0L:24D birds had the greatest intensity of wing flapping. The 24L:0D and the

252 12L:12D birds wing flapped less intensely than the $0 \mathrm{~L}: 24 \mathrm{D}$ birds $(\mathrm{P}=0.05, \mathrm{P}=0.001$,

253 respectively). In Experiment 2, the 0L:24D birds wing flapped more intensely than all

254 other birds $(\mathrm{P}<0.01)$. There were no differences between treatments in number of wing

255 flaps, duration of wing flapping, percent of birds body curling, or \% of birds vocalizing in

256 either experiment (Table 2, P > 0.05).

257 3.3. Approach and Emergence Tests

258 Data from APPR and EMRG are presented in Figure 1. Only distress calls were 259 given, and 12L:12D birds vocalized less $(\mathrm{P}=0.02)$ than 0L:24D birds. They were also 260 less active $(\mathrm{P}=0.05)$, and spent more time in the area closest to the observer $(\mathrm{P}=0.02)$ 261 than the 0L:24D birds. Treatment also affected latency to emerge from the box $\left(\mathrm{F}_{3,336}=\right.$ 262 4.24, $\mathrm{P}=0.006$, Table 1$)$, with the $12 \mathrm{~L}: 12 \mathrm{D}$ birds having a shorter $(\mathrm{P}<0.03)$ latency to 263 emerge than all other treatments. The 12L:12D and 6L:18D birds turned right (0.68 and 
2640.52 right, respectively) when exiting the box significantly more than expected by chance

265 (chance score $=0, \mathrm{P}<0.0001$ ), whereas the $1 \mathrm{~L}: 23 \mathrm{D}$ and $0 \mathrm{~L}: 24 \mathrm{D}$ birds turned left or

266 right at chance levels ( $\mathrm{P}>0.05,0.11$ and 0.12 right, respectively). There was no

267 interaction between treatment and testing day $(\mathrm{P}>0.05)$.

268 4. Discussion

269 Our experiments indicate that light stimulation during incubation is important for 270 reducing fearfulness in 3-6 week old broiler chickens. Providing at least 12 hours of light

271 daily throughout incubation resulted in consistent decreases in fearfulness as evidenced

272 by a decreased latency to right in the tonic immobility test, less intense wing flapping in

273 the inversion test, less distress calling and more proximity to the observer in the approach

274 test, and a shorter latency to leave the box in the emergence test. Given that chicken

275 embryos are typically incubated commercially in complete darkness, these results have

276 important implications for welfare, since excessive or prolonged fear can result in wasted

277 energy, injuries, behavioral inhibition, reduced ability to adapt to change, delayed

278 maturation, decreased growth and reproduction, and death (Jones, 1986). In general, our

279 results are consistent with those of previous studies examining the effects of light

280 stimulation during embryogenesis on fearfulness in domestic fowl. Light incubated

281 chicks have been reported to give fewer distress calls and interrupt feeding behavior more

282 in response to a simulated predator than chicks incubated in darkness (Dharmaretnam and

283 Rogers, 2005; Wichman et al., 2009). They also have shorter latencies to approach novel

284 stimuli (Adam and Dimond, 1971; Deng and Rogers, 2002). However, these previous

285 studies were all conducted on chicks that were 3-9 days old and so could be measuring

286 relatively short-term effects of incubation conditions. To our knowledge, no other studies 
287 have examined whether light stimulation during incubation results in long-lasting effects

288 on fearfulness. The persistency of these responses, as well as their consistency across

289 four different fear tests involving human handling or proximity, have important practical

290 implications. They suggest that providing appropriate light stimulation during incubation

291 could potentially reduce the fear responses of broilers to caretakers and during hand-

292 catching prior to slaughter in the commercial setting.

293 We measured the direction of exit in the emergence test to determine if there was

294 a relationship between reduced fearfulness and laterality. There is growing evidence

295 across taxa that responses associated with fear, and particularly anti-predator responses,

296 are lateralized (Bisazza et al., 1998), with populations or individuals showing side biases

297 for aspects of anti-predator behavior such as vigilant gaze or escape direction (reviewed

298 in Leliveld et al., 2013).

299 The visual pathways in avian embryos are known to become lateralized as a result

300 of the positioning of the embryo's head during the last four days of incubation (Rogers,

301 1982). At this time, the head turns so that the right eye is facing outwards towards the

302 shell and thus receives light stimulation when the parents leave the nest. This stimulates

303 the development of the neural pathways associated with that eye (Rogers, 1996; Rogers

304 and Deng, 1999; Koshiba et al., 2002) and results in lateralization of the two cerebral

305 hemispheres. Light stimulation during early embryogenesis, before the development of a

306 functional visual system, can also result in cerebral lateralization, possibly via changing

307 gene expression in photosensitive regions (Chiandetti et al., 2013). Although there are

308 differences in patterns across taxa, this lateralization results in each hemisphere becoming 
309 dominant for certain functions, including processing of fear-inducing stimuli (Leliveld et

310 al, 2013).

311 In our second experiment, the 6:L18D and 12L:12D birds both showed evidence

312 of laterality in terms of directionality of exit from the dark box. However, fear reactions

313 were only reduced consistently in the 12L:12D birds, suggesting that additional

314 mechanisms could underlie the responses we observed. Other possibilities include the

315 effects of incubation lighting on patterns of hormone secretion associated with the

316 establishment of circadian rhythms (Zeman et al, 1992; Hill et al., 2004), or epigenetic

317 influences (Keverne and Curley, 2008).

\section{5. Conclusions}

319 Our results demonstrate that light stimulation during incubation of chicken embryos

320 has long-lasting effects on anti-predator fear responses, with at least 12 hours of light

321 stimulation required daily. While the exact mechanism underlying these effects is

322 unknown, this research provides evidence that providing an appropriate lighting

323 environment during incubation could improve the welfare of broiler chickens by reducing

324 fearfulness towards humans post-hatch. Future studies could also examine the effects of

325 incubation lighting on laying hens, since panic due to excessive fearfulness in

326 commercial non-cage flocks can be a significant cause of mortality 


\section{References}

Adam, J. , Dimond S. J.,1971. The effect of visual stimulation at different stages of embryonic development on approach behaviour. Animal Behaviour, 19, 51-54.

Archer, G.S., Shivaprasad, H.L , Mench, J.A., 2009. Effect of providing light during incubation on the health, productivity, and behavior of broiler chickens. Poult. Sci. 88, 29-37.

Archer, G. S., Mench, J.A., 2014. The effects of the duration and onset of light stimulation during incubation on the behavior, plasma melatonin levels, and productivity of broiler chickens. J. Anim. Sci. 92, 1753-1758.

Bertin, A., Calandreau, L., Arnould, C., Nowack, R., Levy, F., Noitott, V., Bouvarel, I. , Leterrier, C., 2010. In ovo olfactory experience influences post-hatch feeding behavior in young chickens. Ethology, 116,1027-1037.

Bisazza, A., Rogers, L.J., Vallortigara, G., 1998. The origins of cerebral asymmetry: a review of evidence of behavioural and brain lateralization in fishes, reptiles, and amphibians. Neuroscience and Biobehavioral Reviews, 22, 411-426.

Buschmann, J.U.F., Manns, M., Güntürkün, O., 2006. “Let There be Light!” pigeon eggs are regularly exposed to light during breeding. Behavioural processes 73:62-67.

Chiandetti, C., Galiussi, J., Andrew, R.J., Vallortiagar, G., 2013. Earliy-light embryonic stimulation suggests a second route, via gene activation, to cerebral lateralization in vertebrates. Scientific Reports, 3, doi:10.1038/srep02701.

Cooper, C. B., Voss, M.A., Ardia, D.R., Austin, S.H., , Robinson, W.D., 2011. Light increases the rate of embryonic development: implications for latitudinal trends in incubation period. Functional Ecology 25,769-776.

Deng, C., Rogers, L. J., 2002. Social recognition and approach in the chick: lateralization and effect of visual experience. Animal Behaviour, 63, 697706.

Dharmaretnam, A., Rogers, L. J., 2005. Hemispheric specialization and dual processing in strongly versus weakly lateralized chicks. Behavioural Brain Research, 162, 62-70.

Dimond, S. J., 1968. Effects of photic stimulation before hatching on the development of fear in chicks. J. Comparative Physiological Psychology, 65, 320-324.

FASS, 2010. Guide for the Care and Use of Agricultural Animals in Agricultural Research and Teaching. Federation of Animal Science Societies, Savoy, Il.

Gottlieb, G., 1985. Development of species identification in ducklings: XI. Embryonic critical period for species-typical perception in the hatchling. Animal Behaviour, 33, 225-233.

Groothuis, T. G. G., Müller, W., von Engelhardt, N., Carere, C., Eising, C. M., 2005. Maternal hormones as a tool to adjust offspring phenotype in avian species. Neuroscience Biobehavior Review, 29, 329-352.

Hill, W. L., Bassi, K. L., Bonaventura, L. , Sacus, J. E., 2004. Prehatch entrainment of circadian rhythms in the domestic chick using different light regimes. Developmental Psychobiology, 45, 174-186.

Jones, R.B., 1986. The tonic immobility reactions of the domestic-fowl -a review. World's Poultry Science Journal, 42, 82-96.

Keverne, E. B., Curley, J. P., 2008. Epigenetics, brain evolution and behaviour. 
Frontiers Neuroendocrinology, 29, 398-412.

Koshiba, M., Kikuchi, T., Yohda, M., Nakamura, S., 2002. Inversion of the anatomical lateralization of chick thalamofugal visual pathway by light experience. Neuroscience Letters, 318, 113-116.

Leliveld, L.M.C., Langbein, J., Puppe, B., 2013. The emergence of emotional lateralization: Evidence in non-human vertebrates and implications for farm animals. Applied Animal Behaviour Science, 145, 1-14.

Miller, K. A., J. P. Garner, J. P. , Mench, J. A., 2005. The test-retest reliability of Four behavioural tests of fearfulness for quail: a critical evaluation. Applied AnimalBehaviour Science, 92,113-127.

Miller, K. A., J. P. Garner, J. P.,Mench, J. A., 2006. Is fearfulness a trait that can be measured with behavioural tests? A validation of four fear tests for Japanese quail. Animal Behaviour, 71, 1323-1334.

Mrosovsky, N., Sherry, D. F.,,1980. Animal anorexias. Science, 207, 837-842.

Newberry, R. C., Blair, R., 1993. Behavioral-responses of broiler-chickens to handling - effects of dietary tryptophan and 2 lighting regimens. Poultry Science, 72, 1237-1244.

Phillips, R. E., Youngren, O. M., 1986. Unilateral kainic acid lesions reveal dominance of right archistriatum in avian fear behavior. Brain Research, 377, 216-220.

Reed, W. L., Clark, M. E. , 2011. Beyond maternal effects in birds: responses of the embryo to the environment. Integrative and comparative biology 51, 73-80.

Riedstra, B., Groothuis, T.G.G., 2004. Prenatal light exposure affects early featherpecking behavior in the domestic chick. Animal Behaviour, 6, 1037-1042.

Rogers, L. J. 1982. Light experience and asymmetry of brain-function in chickens. Nature, 297, 223-225.

Rogers, L. J.,. Workman, L., 1989. Light exposure during incubation affects competitive behavior in domestic chicks. Applied Animal Behaviour Science, 23, 187-198.

Rogers, L. J.,1990. Light input and the reversal of functional lateralization in the chicken brain. Behavioural Brain Research, 38, 211-221.

Rogers, L. J. ,1996. The Development of Brain and Behaviour in the Chicken. Wallingford, Oxon: CABI Publishing.

Rogers, L. J., Deng, C., 1999, Light experience an lateralization of the two visual pathways in the chick. Behavioural Brain Research, 98, 277-287.

Rogers, L. J., 2008. Development and function of lateralization in the avian brain. Brain Research Bulletin, 76, 235-244.

Sui, N., Rose, S.P.R.,1997. Effects of dark rearing and light exposure on memory for a passive avoidance task in day-old chicks. Neurobiology of Learning and Memory, 68, 230-238.

Wichman, A., Freire, R., Rogers, L.J., 2009, Light exposure during incubation and social and vigilance behaviour of domestic chicks. Laterality 14:381-394.

Zeman, M., Gwinner, E., Somogyiova, E., 1992. Development of melatonin rhythm in the pineal-gland and eyes of chick-embryo. Experientia, 48, 765-768. 
431 This research was funded by USDA grant \#2005-35204-16110 USDA-CSREES and

432 grant \#2008-35204-19395 USDA-CSREES. We acknowledge and thank Jackie Psienti and

433 Amanda Grout for their assistance with the animal care. We also thank Foster Farms and Cobb-

434 Vantress, Inc. for supplying the eggs used in this study.

435

436

437

438

439

440

441

442

443

444

445

446

447

448

449

450

451

452

453

454

455

456

457

458

459

460

461

462

463

464

465

466

467

468

469

470 
Table 1

Measures with treatment effects for the Tonic Immobility and Inversion tests in Experiments 1 and 2 (means $\pm \mathrm{SE}$ ). Asterisks indicate significant differences between the light treatment groups (1L:23D, 6L:18D, 12L:12D, 24L:0D) and dark-incubated birds (OL:24D)

$\begin{array}{cc}\text { Tonic Immobility } & \text { Inversion } \\ \text { Latency to right (sec) } & \text { Intensity (wing flaps/sec) }\end{array}$

\section{Experiment 1}

\begin{tabular}{ccc}
\hline 0L:24D & $305.5 \pm 26.1$ & $5.7 \pm 0.1$ \\
12L:12D & $213.5 \pm 23.7 * *$ & $5.0 \pm 0.1^{* * *}$ \\
24L:0D & $231.8 \pm 24.2^{*}$ & $5.4 \pm 0.2^{*}$ \\
& \multicolumn{2}{c}{ Experiment 2} \\
\hline 0L:24D & $201.4 \pm 24.9$ & $5.5 \pm 0.1$ \\
1L:23D & $218.2 \pm 21.9$ & $5.1 \pm 0.1^{* *}$ \\
6L:18D & $193.7 \pm 20.8$ & $4.9 \pm 0.1^{* * *}$ \\
12L:12D & $120.0 \pm 16.5^{* *}$ & $4.7 \pm 0.1^{* * * *}$ \\
\hline
\end{tabular}

$* \mathrm{P}<0.05 ; * * \mathrm{P}<0.01 ; * * * \mathrm{P}<0.001 ; * * * * \mathrm{P}<0.0001$ 
Table 2

Measures with no treatment effects for the Tonic Immobility and Inversion tests in Experiments 1 and 2 (means \pm SE)

\begin{tabular}{|c|c|c|c|}
\hline \multirow{3}{*}{ Tonic Immobility } & & Experiment 1 & Experiment 2 \\
\hline & $\begin{array}{c}\text { Induction } \\
\text { attempts (\#) }\end{array}$ & $1.2 \pm 0.06$ & $1.3 \pm 0.06$ \\
\hline & $\begin{array}{l}\text { Latency to } \\
\text { first head } \\
\text { movement } \\
\quad \text { (sec) }\end{array}$ & $24.1 \pm 3.1$ & $49.2 \pm 10.0$ \\
\hline \multirow[t]{4}{*}{ Inversion } & $\begin{array}{c}\text { Duration of } \\
\text { wing flapping } \\
\text { (sec) }\end{array}$ & $5.7 \pm 0.3$ & $5.9 \pm 0.3$ \\
\hline & Wing flaps (\#) & $30.7 \pm 1.6$ & $29.0 \pm 1.2$ \\
\hline & $\begin{array}{c}\text { Vocalization } \\
\text { (\% birds) }\end{array}$ & $9 \pm 1$ & $9 \pm 1$ \\
\hline & $\begin{array}{l}\text { Body curls } \\
\text { (\% birds) }\end{array}$ & $88 \pm 2$ & $90 \pm 2$ \\
\hline
\end{tabular}


508 Figure 1. Results from the approach (\# Vocalizations, \% Time spent active, and \%S 509 time closest to the observer) and emergence (Latency to exit box) tests. *Indicates 510 significantly different from 0L:24D treatment $(\mathrm{P}<0.05)$. 